\title{
La evaluación bioquímica de la proteinuria de caninos en el laboratorio de análisis clínicos veterinarios
}

\section{The biochemical evaluation of canine proteinuria in the veterinary clinical analysis laboratory}

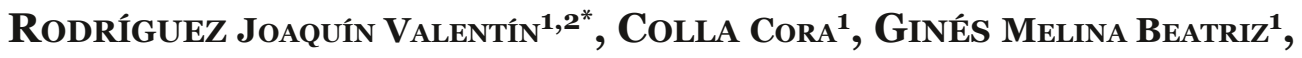 \\ SCHRÖDER GISEL ${ }^{1}$
}

1. Laboratorio Centralizado. Hospital Escuela de Grandes y Pequeños Animales, (HEGYPA). Facultad de Ciencias Veterinarias. 2. Centro Binacional de Investigaciones en Criobiología Clínica y Aplicada (CAIC). Universidad Nacional de Rosario (UNR). Argentina.

* Correo electrónico del autor de contacto: jrodrig@fbioyf.unr.edu.ar

\begin{abstract}
Resumen
Es sabido que la proteinuria en perros y gatos está asociada a morbilidad renal y que puede ser utilizada como marcador de riesgo de la evolución de enfermedad renal y también de la respuesta a tratamientos curativos. Existe una controversia acerca de los valores que la proteinuria puede alcanzar en perros sanos. El objetivo de este estudio fue establecer los límites de las concentraciones de las proteínas urinarias que pueden presentarse en caninos sanos utilizando métodos analíticos disponibles en el laboratorio de análisis clínicos veterinarios. La interpretación de los resultados obtenidos en el análisis de orina requiere la normalización de los mismos respecto del estado de hidratación del paciente. Por esta razón se normalizan los datos obtenidos de proteinuria a la concentración de creatinina urinaria. La relación proteína/creatinina indica entonces la concentración de proteína urinaria normalizada al estado de hidratación del paciente. Se analizaron 74 muestras de orina provenientes de animales clínicamente sanos y de otros con diversas enfermedades, los que fueron agrupados sobre la base de una proteinuria < $300 \mathrm{mg} / \mathrm{l}$ (45 muestras) y proteinuria $>300 \mathrm{mg} / \mathrm{l}$ (29 muestras). En todas las muestras se determinó la relación concentración de proteínas/concentración de creatinina. Los resultados mostraron que las proteinurias de hasta $300 \mathrm{mg} / \mathrm{l}$ asociadas a una relación proteína/creatinina menor a o,30 no necesariamente indican una proteinuria patológica.
\end{abstract}

\section{Palabras clave}

Caninos, orina, osmometría, proteinuria, refractometría

\begin{abstract}
Proteinuria in dogs and cats is associated with renal morbidity and can be used as a risk marker for the evolution of renal disease and response to treatments. However, there is a controversy about proteinuria values in healthy dogs. The objective of this study was to determine urinary protein concentration values that can occur in healthy dogs using analytical methodology available in the veterinary clinical analysis laboratory. Interpretation of results obtained in the urinalysis requires normalization regarding the patient's hydration status. For this reason the proteinuria data obtained were normalized to the concentration of urinary creatinine (protein/creatinine ratio). Seventy four urine samples from healthy animals and animals with different diseases were analyzed. Animals were grouped based on proteinuria $<300 \mathrm{mg} / \mathrm{l}$ ( 45 samples) and proteinuria $>300 \mathrm{mg} / \mathrm{l}$ ( 29 samples). The protein/creatinine ratio was determined in all samples. Results showed that proteinuria up to $300 \mathrm{mg} / \mathrm{l}$ associated with a protein/creatinine ratio of less than 0.300 does not necessarily indicate a pathological proteinuria.
\end{abstract}

\section{Key words}

Dogs, refractometry, osmometry, proteinuria, urine

Fecha de recepción: 26/07/2019

Fecha de revisión: 22/10/2019

Fecha de aprobación: 16/12/2019
ANALECTA VeT 2020; Enero-Junio; 40(1):33-37

Impresa ISSN 03655 14-8 Electrónica ISSN 1514-2590

doi.org/10.24215/15142590e044 


\section{Introducción}

La proteinuria (PU) como parámetro bioquímico aplicado al diagnóstico es la presencia de una cantidad anormal de proteínas en la orina. Es sabido que la orina de perros sanos contiene pequeñas cantidades de proteínas. La PU persistente se asocia a enfermedad renal (Lees et al., 2005) y la determinación de los valores de la misma debe ser considerada en la formulación de un pronóstico (International Renal Interest Society guidelines, 2019). Existe una controversia acerca de los valores de la PU que pueden observarse en perros clínicamente sanos (Tvedten, 2016). Por lo tanto es de especial interés establecer los límites de las concentraciones de las proteínas urinarias que pueden presentarse en caninos sanos, mediante la metodología analítica disponible en el laboratorio de análisis clínicos veterinarios. En este contexto, sabemos que la concentración de metabolitos urinarios está fuertemente influenciada por el estado de hidratación del paciente y que, particularmente en el caso de caninos, es difícil de controlar en el paciente ambulatorio. La determinación de la creatinina urinaria es de particular interés en la estimación de la concentración de metabolitos urinarios, ya que la misma puede ser utilizada como una medida de la concentración de la orina (Waldrop, 2008). Por ello es necesario introducir, en el análisis de datos, un factor de corrección. Esto se realiza normalizando la concentración del metabolito, en este caso la concentración de proteínas totales urinarias $(\mathrm{P})$ a la concentración de la creatinina urinaria (C). La relación $\mathrm{P} / \mathrm{C}$ indica entonces la concentración de proteínas totales urinarias normalizada al estado de hidratación del paciente. El objetivo de este estudio fue establecer los límites de las concentraciones de las proteínas urinarias que pueden presentarse en caninos clínicamente sanos utilizando métodos analíticos disponibles en el laboratorio de análisis clínicos veterinarios.

\section{Descripción metodológica}

Se analizaron 74 muestras de orina provenientes de un universo de pacientes caninos comprendidos en alguno de los siguientes criterios de inclusión: 1- perros de distintas edades (1 a 10 años), sexo, raza y peso corporal. 2- animales clínicamente sanos cuyos análisis urinarios fueron realizados durante controles de salud. 3- perros con diferentes enfermedades, con y sin compromiso renal metabólico e inflamatorio, potencialmente asociadas a PU.

Las muestras fueron obtenidas por micción espontánea en su mayoría o por cistocentesis $\mathrm{y}$ fueron recibidas y procesadas en el laboratorio. En todas las muestras se determinaron los siguientes parámetros: 1- Densidad urinaria (DU) mediante refractometría (refractómetro clínico Alla
France mod. 95000-017). 2- Osmolalidad urinaria (OSM) mediante la técnica de descenso crioscópico (Osmomat 030, Gonotec). Para calibrar el instrumento se utilizaron stándares de 300,850 y 2.00O mOsm/ $/ \mathrm{Kg} \mathrm{H}_{2} \mathrm{O}$. 3- Parámetros bioquímicos urinarios obtenidos utilizando tiras reactivas (TR) (Siemens Multistix 1056). Los mismos fueron estimados por un solo operador, por comparación colorimétrica visual con la escala provista en el kit. 4- Proteínas totales urinarias (P) mediante el método espectrofotométrico del rojo de pirogalol (Proti U/LCR, Wiener Lab, Argentina). 5Creatinina urinaria (C) mediante el método de Jaffé (Biggs \& Cooper, 1961) después de la dilución de las muestras con agua destilada (1/10 o $1 / 50$ en relación a la DU) (Rossi et al., 2012). El procedimiento analítico realizado en todas las muestras fue el descripto por Rodríguez et al. (2018): en primer lugar se determinaron los parámetros bioquímicos urinarios mediante TR y luego la DU; posteriormente, las muestras fueron conservadas a $-20{ }^{\circ} \mathrm{C}$ hasta la determinación de OSM, P y C. Para todas las muestras se calculó la relación P/C. En las orinas que contenían concentraciones considerables de proteínas se realizó la siguiente corrección de la DU (Rodríguez et al., 2018): por cada $\mathrm{g} / \mathrm{l}$ de proteína urinaria, se sustrajeron 0,003 unidades a la DU medida mediante RF. En los casos en que la DU excedió los límites establecidos en el refractómetro (DU > $1,050)$, las muestras fueron diluidas al medio (1/2) con agua destilada y luego analizadas. Para determinar la DU de la dilución se multiplicó la porción decimal de la nueva lectura por la dilución realizada (Sink \& Weinstein, 2012), por ejemplo: DU determinada en la dilución $1 / 2=1,020$, luego $0,020 \times 2=0,040$, obteniéndose, entonces, la DU corregida que fue de 1,040. Si bien no se incluyen en este trabajo los resultados de los sedimentos urinarios, estos fueron utilizados para confirmar diagnósticos clínicos. Así, por ejemplo, fueron excluidos del grupo de animales clasificados como clínicamente sanos, aquellos que pre-sentaron piuria o hematuria en los estudios del sedimento urinario.

\section{Resultados y Discusión}

Para facilitar el análisis de los datos, se decidió agruparlos de la siguiente manera: grupo 1: orinas que presentaron concentraciones de $\mathrm{P}<$ $300 \mathrm{mg} / \mathrm{l}$ (45 muestras) y grupo 2: orinas que presentaron P > $300 \mathrm{mg} / \mathrm{l}$ (29 muestras) (en este grupo se incluyeron dos muestras cuyas $\mathrm{P}$ estuvieron en el rango de 276 a $300 \mathrm{mg} / \mathrm{l}$ y sus $\mathrm{P} / \mathrm{C}$ eran > $0,30)$.

Para el grupo 1, las DU presentaron un intervalo de valores de 1,004 a 1,058 (media 1,028) y OSM de 118 a $2116 \mathrm{mOsm} / \mathrm{KgH}_{2} \mathrm{O}$ (media 1063). En algunas muestras, las determinaciones de bioquímica urinaria realizadas mediante TR 
indicaron valores de $\mathrm{P}$ que variaron desde trazas a $1+(100-300 \mathrm{mg} / \mathrm{l}$ de acuerdo con las instrucciones del fabricante). En las figuras $1 \mathrm{~A}$ y $1 \mathrm{~B}$ se muestran las concentraciones de $\mathrm{P}$ ordenadas de menor a mayor con sus correspondientes relaciones $\mathrm{P} / \mathrm{C}$.

En la figura $1 \mathrm{~A}$ pueden observarse las concentraciones de $\mathrm{P}$ cuyos valores oscilaron desde 14 a 288 mg/l (media 127). La relación P/C en este grupo fue variable y se distribuyó en un rango de valores < 0,30 (mínima 0,019 - máxima 0,29). Se destaca que el aumento de las concentraciones de $\mathrm{P}$ no se asoció con un aumento de la relación $\mathrm{P} / \mathrm{C}$. Para el grupo 2, las DU, corregidas por la concentración de $\mathrm{P}$, presentaron un intervalo de valores de 1,002 a 1,040 y una OSM de 108 a 1474 mOsm $/ \mathrm{KgH}_{2} \mathrm{O}$ (media 805). En todas estas muestras las TR mostraron concentraciones de P desde 1 a $3^{+}$. En la figura $1 \mathrm{~B}$ se presentan las concentraciones de $\mathrm{P}$ correspondientes al grupo 2, cuyos valores oscilaron desde 276 a $7350 \mathrm{mg} / \mathrm{l}$ (media 1365). Es importante considerar la diferencia de escalas presentadas en la figura $1 \mathrm{~B}$ respecto de la figura $1 \mathrm{~A}$ (6 veces mayor para $\mathrm{P} / \mathrm{C} \mathrm{y}$ hasta $8000 \mathrm{mg} / \mathrm{l}$ para $\mathrm{P})$. Puede entonces determinarse un comportamiento diferente $\mathrm{y}$ disperso de la relación $\mathrm{P} / \mathrm{C}$ que se incrementa hasta valores mayores a 5,5 (mínima 0,216 máxima 5,66). En este grupo, la relación $\mathrm{P} / \mathrm{C}$ de algunas orinas manifestó un incremento asociado al aumento de las concentraciones de P. Este grupo de caninos se correspondió al de animales con varias enfermedades.

Considerando los posibles efectos de la hidratación del paciente sobre los valores de la $\mathrm{C}$ nos pareció importante estudiar la relación existente entre la $\mathrm{C}$ y la osmolalidad urinaria en ambos grupos de caninos.

En la figura 2 se presentan las relaciones entre la osmolalidad y la concentración urinaria de creatinina de las muestras de orina analizadas, a las que se aplicó el análisis de regresión y correlación de ambas variables. La figura $2 \mathrm{~A}$ muestra los datos correspondientes a animales sanos cuya relación $\mathrm{P} / \mathrm{C}$ fue $<0,30$. Podemos observar una fuerte asociación entre ambas variables en este grupo. En cambio, en la figura 2B (animales con varias enfermedades y relación $\mathrm{P} / \mathrm{C}>0,30$ ) la asociación entre variables fue muy pobre, lo cual indica que el aumento de $\mathrm{P}$ en estos casos no está relacionado con el estado de hidratación del paciente.

Consideramos que el análisis de 45 muestras provenientes de caninos clínicamente sanos, constituye una cantidad adecuada para establecer la estimación de un nuevo intervalo de referencia en el análisis de la $\mathrm{P}$ en nuestro ámbito. Las concentraciones de $\mathrm{P}$ deben ser interpretadas considerando la concentración de la orina; por ello es importante asociarla a la concentración de creatinina urinaria, ya que la relación $\mathrm{P} / \mathrm{C}$ normaliza el valor de la PU a la concentración o dilución de la orina (Trumel et al., 2004). Tvedten (2016) ha informado, en perros sanos, valores de $\mathrm{P}$ que oscilaron desde 30 a $390 \mathrm{mg} / \mathrm{l}$ y relaciones $\mathrm{P} / \mathrm{C}$ ligeramente menores a las obtenidas por nosotros (0,02 - 0,21) vs (0,019 - 0,29). En ambos parámetros no existen diferencias substanciales con las observadas en nuestro laboratorio. Por otra parte, Rossi et al. (2016) han señalado la importancia del método de determinación de $\mathrm{P}$ y su posible efecto en los intervalos de referencia sobre proteinuria (International Renal Interest Society guide-lines, 2019). En este trabajo, la relación $P / C$ se incluye en el límite inferior del grupo borderline (0,200,50) de las guías IRIS (2019), las cuales establecen una proteinuria $<0,2$ como no proteinúrica. El intervalo (0,019 - 0,29) es mayor y podría estar asociado a alguna diferencia metodológica, tanto en el método usado para la determinación de proteínas como en la determinación de creatinina.

A

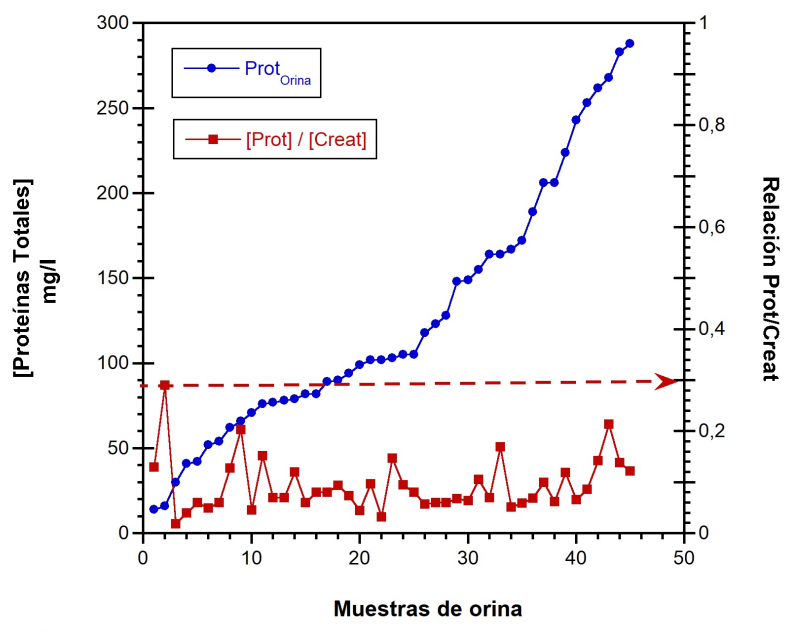

B

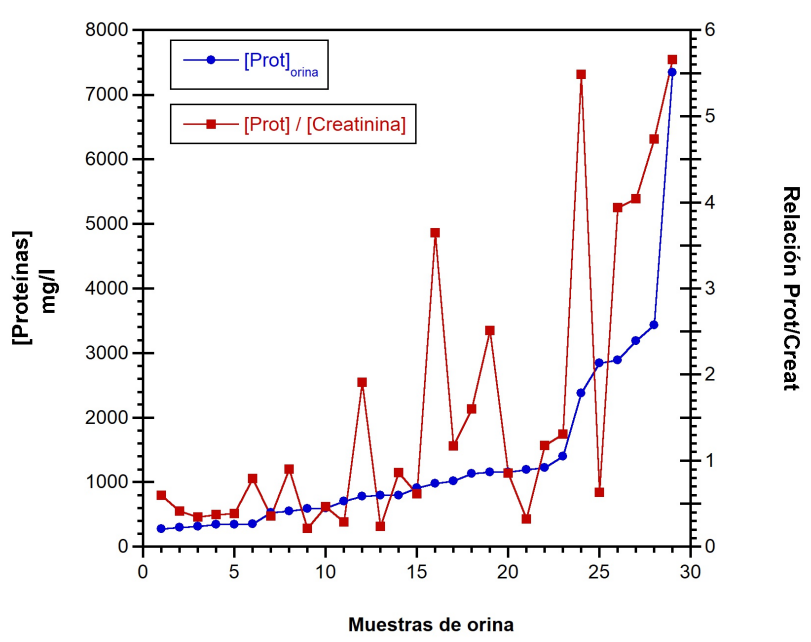

Figura 1. A- Concentración de proteína total urinaria y relación proteína/creatinina en orinas cuya concentración de proteínas es $<300 \mathrm{mg} / \mathrm{l}$ ( $\mathrm{n}=45$ muestras). B- Concentración de proteína total urinaria y relación proteína/creatinina en orinas cuya concentración de proteínas es > 300 mg/l ( $\mathrm{n}=29$ muestras). 
Respecto del método utilizado para la determinación de proteínas en estudios similares, no estamos de acuerdo con la utilización de otras técnicas que utilizan colorantes como el azul brillante de Comassie, por sus desventajas analíticas, por ejemplo su adsorción sobre tubos plásticos, etc. (Goren et al., 1985; Iijima et al., 2003; Shahangian et al., 1986). El método del rojo de pirogalol, con las modificaciones sugeridas por otros autores debidas a posibles interferencias (Marshall \& Williams, 2003; Marshall \& Williams, 2004) y realizadas por los fabricantes de los kits disponibles en nuestro medio, constituye, en nuestra apreciación, un método simple, económico y bastante exacto, considerando la complejidad de estimar la concentración de proteínas totales en un líquido biológico como la orina. Por lo expuesto, podemos concluir que, en orinas de caninos de nuestro medio, la presencia de trazas de $\mathrm{P}(1+)$, detectadas mediante TR, o concentraciones $<300$ $\mathrm{mg} / \mathrm{l}$, determinadas mediante la reacción del rojo de pirogalol, asociada a una relación $\mathrm{P} / \mathrm{C}<0,30$, no indican necesariamente una proteinuria patológica.

A

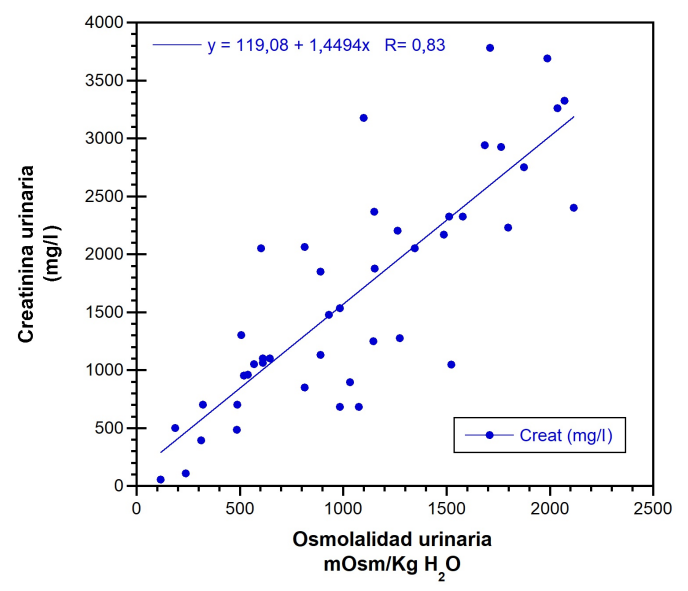

B

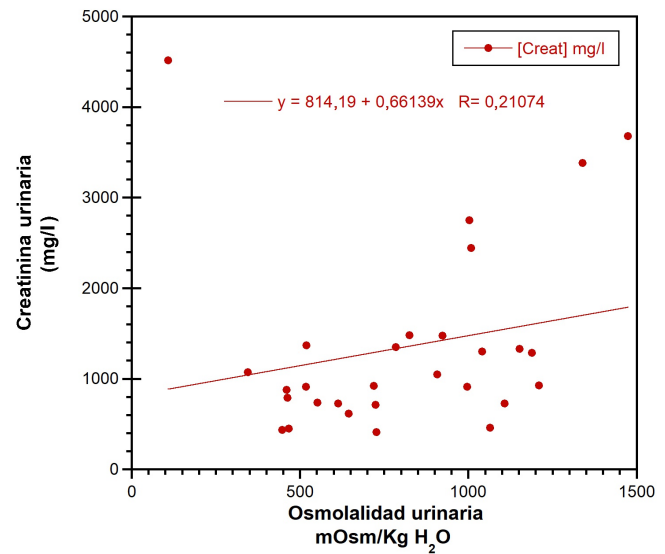

Figura2. A- Concentración de creatinina en orina de caninos sanos en función de la osmolalidad urinaria ( $n=45$ muestras). B- Concentración de creatinina en orina de caninos con proteinuria en función de la osmolalidad urinaria $(n=29$ muestras).

\section{Agradecimientos}

Agradecemos a Wiener Lab., por la generosa donación del Kit Proti U/LCR. Los instrumentos utilizados fueron provistos por el subsidio del Servicio de Relaciones Internacionales y Partenariado Territorial de la Región FriuliVenezia-Giulia de Italia para la ejecución del proyecto Creación del Centro Binacional (Argentina - Italia) de Investigación en Criobiología Clínica y Aplicada. Protocolo: n. 19096/PT.

\section{Conflicto de intereses}

Todos los autores declaran que no existe conflicto de intereses, relaciones financieras, personales o de otro tipo con personas u organizaciones que pudieran afectar al presente trabajo.

\section{Bibliografía}

Biggs HG, Cooper JM. 1961. Modified Folin methods for the measurement of urinary creatine and creatinine. Clinical Chemistry. 7(6):655-64. doi: 10.1093/clinchem/7.6.655

Goren MP, Wright RK, Li JT. 1985. Total protein concentrations determined for CAP urine survey specimens with Coomassie Brilliant Blue reagent are dilution dependent. Clinical Chemistry. 31(10): 1771-2.

Iijima S, Cho H, Sakai N, Shiba K, Toyoshima Y, Nishida K, Kobayashi S. 2003. Development of a new method for measuring total urinary protein using acid violet 6B pigment. Journal Clinical Laboratory Analysis. 17(5):147-54.

doi:10.1002/jcla.10085

International Renal Interest Society (IRIS) guidelines: IRIS staging of CDK, 2019. Disponible en:

http://www.iriskidney.com/pdf/

IRIS_Staging_of_CKD_modified_2019.pdf.

[Consultado noviembre 13, 2019].

Lees GE, Brown SA, Elliott J, Grauer GF, Vaden SL. 2005. Assessment and management of proteinuria in dogs and cats: 2004 ACVIM Forum Consensus Statement (Small Animal). Journal of Veterinary Internal Medicine. 19(3):377-85.

doi:10.1892/o891-

6640(2005)19[377:aamopi]2.o.co;2

Marshall T, Williams KM. 2003. Total protein determination in urine: aminoglycoside interference. Clinical Chemistry. 49(1):202-3.

doi: 10.1373/49.1.202 
Marshall T, Williams KM. 2004. Elimination of the interference from aminoglycoside antibiotics in the pyrogallol red-molybdate protein dye-binding assay. Clinical Chemistry. 50(9):1674-5.

doi: 10.1373/clinchem.2004.036392

Rodríguez JV, Colla C, Ginés MB, Schröder G. 2018. Determinación de la concentración de solutos en orinas de pacientes caninos: comparación de osmometría versus densidad urinaria (refractometría y tiras reactivas). Analecta Veterinaria. 38(1):45-9.

doi: 10.24215/15142590eo24

Rossi G, Giori L, Campagnola S, Zatelli A, Zini E, Paltrinieri S. 2012. Evaluation of factors that affect analytic variability of urine protein-to-creatinine ratio determination in dogs. American Journal of Veterinary Research. 73(6):779-88.

doi: 10.246o/ajvr.73.6.779

Rossi G, Bertazzolo W, Binella M, Scarpa P, Paltrinieri S. 2016. Measurement of proteinuria in dogs: analytic and diagnostic differences using 2 laboratory methods. Veterinary Clinical Pathology. 45(3):450-8. doi: 10.1111/vcp.12388
Shahangian S, Brown PI, Ash KO. 1986. More on the dilution dependence of the Coomassie Brilliant Blue technique for urinary protein. Clinical Chemistry. 32(2):403.

Sink CA, Weinstein NM. 2012. Chapter 3: Routine urinalysis: physical properties. En: Urine practical veterinary urinalysis. West Sussex, UK: WileyBlackwell. pp. 23-4.

Trumel C, Diquélou A, Lefebvre H, Braun JP. 2004. Inaccuracy of routine creatinine measurement in canine urine. Veterinary Clinical Pathology. 33(3):128-32.

doi: 10.1111/j.1939-165X.2004.tboo361.x

Tvedten H. 2016. Urine protein total concentration in clinically normal dogs. Veterinary Clinical Pathology. 45(3):395-6.

doi:10.1111/vcp.12379

Waldrop JE. 2008. Urinary electrolytes, solutes, and osmolality. Veterinary Clinical North America Small Animal Practice. 38(3):503-12.

doi:10.1016/j.cvsm.2008.01.011 